\title{
A NOTE ON BRANCHING RANDOM WALKS ON FINITE SETS
}

\author{
THOMAS MOUNTFORD, ${ }^{*}$ EPFL-DMA \\ RINALDO B. SCHINAZI, ${ }^{* *}$ University of Colorado
}

\begin{abstract}
We show that a branching random walk that is supercritical on $\mathbb{Z}^{d}$ is also supercritical, in a rather strong sense, when restricted to a large enough finite ball of $\mathbb{Z}^{d}$. This implies that the critical value of branching random walks on finite balls converges to the critical value of branching random walks on $\mathbb{Z}^{d}$ as the radius increases to infinity. Our main result also implies coexistence of an arbitrary finite number of species for an ecological model.
\end{abstract}

Keywords: Branching random walk; coexistence; ecological model; spatial stochastic model; contact process

2000 Mathematics Subject Classification: Primary 60K35

\section{Introduction and results}

Consider a branching random walk $\left(\xi_{t}, t \geq 0\right)$ on $\left\{\mathbb{Z}_{+}\right\}^{\mathbb{Z}^{d}}=\{0,1,2, \ldots\}^{\mathbb{Z}^{d}}$. An unbounded number of individuals is permitted at each site. More precisely, for $x \in \mathbb{Z}^{d}$ and $\xi \in\{0,1,2, \ldots\}^{\mathbb{Z}^{d}}, \xi(x)=0$ will represent a vacant site $x$ for configuration $\xi$ and $\xi(x)=$ $n>0$ will represent the presence of $n$ individuals at site $x$.

Individuals die at rate 1 and, at each site, new individuals are born according to the number of individuals that are present at neighboring sites. The system is a spin system in that, at any time $t$, at most one site can change; this change must consist of a change in value (up or down) of precisely 1 . For $\xi(x)=n, n>0$, the up-rate is

$$
c_{+}(x, \xi)=\lim _{t \rightarrow 0} \frac{\mathrm{P}^{\xi}\left(\xi_{t}(x)=n+1\right)}{t}=\lambda_{1} \sum_{\left\{y \in \mathbb{Z}^{d}: x \sim y\right\}} \frac{\xi(y)}{2 d},
$$

where $x \sim y$ means that $y$ is one of the $2 d$ nearest neighbors of $x$. The down-rate is

$$
c_{-}(x, \xi)=\lim _{t \rightarrow 0} \frac{\mathrm{P}^{\xi}\left(\xi_{t}(x)=n-1\right)}{t}=n=\xi(x) .
$$

The process $\xi_{t}$ can be constructed using Harris's graphical construction - see, for example, Pemantle and Stacey (2001, Section 3).

Remark 1. Since we are dealing with unbounded spins (i.e. an unbounded number of individuals is possible at each site), the process will not be defined for all $\xi_{0}$. However, following the methods of Kesten and van den Berg (2000), for example, we can show the existence of a nonexplosive process satisfying the above conditions for $\xi_{0}(x)$ bounded over $x$.

Received 10 November 2003; revision received 8 July 2004.

* Postal address: EPFL-DMA, CH-1015 Lausanne, Switzerland. Email address: thomas.mountford@epfl.ch

** Postal address: Department of Mathematics, University of Colorado, Colorado Springs, CO 80933, USA.

Email address: schinazi@math.uccs.edu 
Let $\left|\xi_{t}\right|=\sum_{y \in \mathbb{Z}^{d}} \xi_{t}(y)$ be the number of particles of $\xi_{t}$ at time $t$ for an initial configuration $\xi_{0}$ with $\left|\xi_{0}\right|<\infty$. Note that if $\left|\xi_{t}\right|=n$, then

$$
\begin{aligned}
& \left|\xi_{t}\right| \rightarrow n+1 \quad \text { at rate } n \lambda_{1}, \\
& \left|\xi_{t}\right| \rightarrow n-1 \quad \text { at rate } n .
\end{aligned}
$$

That is, the process $\left|\xi_{t}\right|$ is a continuous-time (nonspatial) branching process. Clearly, its critical value is 1: starting with one individual, there is a positive probability that the process does not become extinct if and only if $\lambda_{1}>1$.

In this paper, we are concerned with branching random walks restricted to a finite set; births from outside the finite set into the set are not permitted. Let $|\cdot|$ denote the Euclidean norm on $\mathbb{Z}^{d}$ and let

$$
B_{n}=\left\{x \in \mathbb{Z}^{d}:\|x\| \leq n\right\} .
$$

A branching random walk restricted to the set $B_{n}$ is the Markov chain on $\{0,1,2, \ldots\}^{B_{n}}$ with transition rates

$$
\begin{aligned}
& \bar{c}_{+}(x, \xi)=\lambda_{1} \sum_{\left\{y \in B_{n}: x \sim y\right\}} \frac{\xi(y)}{2 d} \\
& \bar{c}_{-}(x, \xi)=c_{-}(x, \xi)
\end{aligned}
$$

for $x \in B_{n}$.

Theorem 1. If $\lambda_{1}>1$ then there exists an integer $n$ such that the branching random walk restricted to $B_{n}$ survives in the following (strong) sense. There exists a function $f_{n}$ on $B_{n}$ such that, for any $\alpha>0$, there exists an $N \equiv N(\alpha, n)$ with the property that if

$$
\xi_{0}(x)>N f_{n}(x) \text { for all } x \in B_{n},
$$

then, with probability at least $1-\alpha$, we have, for any $\delta \in(0,1)$,

$$
\xi_{t}(x)>N(1-\delta) f_{n}(x) \mathrm{e}^{\left(\lambda_{1}-1\right) t / 2} \text { for all } x \in B_{n} \text { and all } t>0 .
$$

Theorem 1 is concerned with the behavior of a branching random walk restricted to a finite set when the unrestricted walk is supercritical. A dual point of view is to examine the local behavior of unrestricted branching random walks. This has been done for continuous-space branching random walks; see, for instance, Engländer and Kyprianou (2004) or Engländer and Pinsky (1999), and the references therein.

We now turn to two applications of Theorem 1.

It is easy to see, by the attractiveness of the systems (see, for example, Liggett (1985)), that the branching random walk restricted to $B_{n}$ has a critical value $\lambda_{\mathrm{c}}^{n}$ such that, starting with a single particle, this process becomes extinct with probability 1 for $\lambda_{1}<\lambda_{\mathrm{c}}^{n}$ and becomes extinct with probability strictly less than 1 for $\lambda_{1}>\lambda_{\mathrm{c}}^{n}$. It is also not difficult to show that $\lambda_{\mathrm{c}}^{n}>1$ (the critical value of the unrestricted branching random walk) and is finite, but an exact computation seems out of the question. This is because the birth rate of a particle depends on where the particle is: near the boundary or inside $B_{n}$. For this process (unlike for the unrestricted process) the critical value depends on the geometry of the space to which the process is restricted. However, as a direct consequence of Theorem 1, we obtain the following result.

Corollary 1. The critical value $\lambda_{\mathrm{c}}^{n}$ of the branching random walk on $B_{n}$ converges to the critical value of the branching random walk on $\mathbb{Z}^{d}$ as $n \rightarrow \infty$. 
Proof. According to Theorem 1, for any $\lambda_{1}>1$ there exists an $n_{0}$ such that there is a positive probability for the branching random walk restricted to $B_{n_{0}}$ to survive. (Using the Markov property of the process, it is easy to see that if the process can survive starting from a particular finite distribution, then it can also survive starting from any nonempty finite distribution). Thus, for any $\lambda_{1}>1$, there exists an $n_{0}$ such that $\lambda_{\mathrm{c}}^{n_{0}} \leq \lambda_{1}$. Also, since the sequence $\left(\lambda_{\mathrm{c}}^{n}\right)_{n \geq 1}$ is decreasing and is bounded below by 1 , we find that it converges to 1 as $n$ goes to infinity. This completes the proof.

Note that Liggett (1999) computed asymptotics for $\lambda_{\mathrm{c}}^{n}$ (as $n$ goes to infinity) for branching random walks on finite trees, but even in that case an exact computation of $\lambda_{\mathrm{c}}^{n}$ seems impossible.

Now, consider a model in which $v$ varieties of species compete for space. Each species gives birth and dies according to a branching random walk. Species $i$ has birth rate $\lambda_{i}$ and death rate 1 (although we could take different death rates as well). There is no bound on the number of individuals per site, but we have at most one species per site. That is, birth attempts on sites that are already colonized by another species are suppressed. This process can be viewed as the process $\left(\xi_{t}=\left(\xi_{t}^{1}, \xi_{t}^{2}, \ldots, \xi_{t}^{v}\right), t \geq 0\right)$, where $\xi_{t}^{i}(x)$ is the number of individuals of type $i$ present at position $x$ at time $t$. The prohibition of multiple species at the same site implies that $\xi_{t}^{i}(x) \xi_{t}^{j}(x)=0$ for each $t \geq 0, x \in \mathbb{Z}^{d}$, and distinct $i, j \in\{1,2, \ldots, v\}$. As before, the process is a spin system and if $\xi^{i}(x)=n \geq 0$ and $\sum_{j \neq i} \xi^{j}(x)=0$, we find that

$$
c_{+}(x, \xi, i)=\lim _{t \rightarrow 0} \frac{\mathrm{P}^{\xi}\left(\xi_{t}^{i}(x)=n+1\right)}{t}=\lambda_{1} \sum_{\left\{y \in \mathbb{Z}^{d}: x \sim y\right\}} \frac{\xi^{i}(y)}{2 d} .
$$

The down-rate (for $\xi^{i}(x)=n>0$ ) is

$$
c_{-}(x, \xi, i)=\lim _{t \rightarrow 0} \frac{\mathrm{P}^{\xi}\left(\xi_{t}^{i}(x)=n-1\right)}{t}=n=\xi^{i}(x) .
$$

If the initial configuration has individuals of all $v$ species, it is easy to see that at time 1 , say, there is a positive probability that $v$ balls of a given radius in $\mathbb{Z}^{d}$ are each occupied by a single species. Moreover, there is a positive probability that each species will occupy a ball with a radius and a number of individuals per site large enough that Theorem 1 is applicable. Since there is a positive probability that every site of each colonized ball will be occupied forever by the same species, there is a positive probability that all $v$ species will coexist forever. This proves the following corollary

Corollary 2. Consider an ecological model with $v$ species, in which each species gives birth and dies according to a branching random walk. Let the birth rates be $\lambda_{i}>1,1 \leq i \leq v$, and let the (common) death rate be 1. Each site can be occupied by at most one species. For any initial configuration containing all $v$ species, there is a positive probability that all species will coexist.

Note that coexistence occurs even if some birth rates are much larger than others. This is in sharp contrast to models in which there is a limit of one individual per site. For such a model, it has been shown that two species may coexist if and only if $\lambda_{1}=\lambda_{2}$ and $d \geq 3$ - see Neuhauser (1992).

\section{Proof of Theorem 1}

To prove Theorem 1, we will use coupling arguments as well as some simple quasi-stationary properties of random walks. Our starting point is the existence of a quasi-stationary distribution 
(defined as an eigenvector corresponding to the largest eigenvalue of the transition matrix) for the simple random walk on a finite, connected subset of $\mathbb{Z}^{d}$ with Dirichlet boundary conditions (i.e. the random walk is killed on exiting the set). The largest eigenvalue for the quasi-stationary distribution tends to 1 as the finite set tends pointwise to $\mathbb{Z}^{d}$. In particular, we obtain the following lemma.

Lemma 1. For all $a>0$, there exists an integer $N_{0}$ such that the largest eigenvalue of the subprobability matrix for the simple random walk on $B_{N_{0}}$ with Dirichlet boundary conditions is greater than $1-a$.

Proof. We refer to Aldous and Fill (2003, Chapter 3, Section 6.5) for details of quasistationary distributions. We consider the sub-Markov chain obtained by killing the simple random walk, starting in $B_{n}$, when it leaves $B_{n}$. For this Markov chain the subprobability matrix $\boldsymbol{P}^{n}$ is simply given (entrywise) by

$$
P_{i j}^{n}= \begin{cases}1 / 2 d & \text { if } i \text { and } j \text { are neighbors in } B_{n}, \\ 0 & \text { otherwise. }\end{cases}
$$

There is a quasi-stationary distribution $f_{i}^{n}, i \in B_{n}$, which (collectively) is an eigenvector of $\boldsymbol{P}^{n}$ corresponding to $\mu(n)$, the largest eigenvalue of this matrix. That is, for each $i \in B_{n}$,

$$
f_{i}^{n}=\mu(n) \sum_{j} P_{j i}^{n} f_{j}^{n}=\frac{\mu(n)}{2 d} \sum_{j} f_{j}^{n},
$$

where, in both cases, the summation is over those $j$ in $B_{n}$ that are neighbors of site $i$.

The eigenvalue $\mu(n)$ is endowed with the following probabilistic meaning:

$$
\mathrm{P}^{i}\left(\tau_{n} \geq N\right) \sim(\mu(n))^{N} \text { for all } i \in B_{n} .
$$

Here, $\tau_{n}$ is the death time of the sub-Markov chain (or, equivalently, the quitting time of $B_{n}$ for the unrestricted simple random walk) and ' $\sim$ ' means that the ratio of the two quantities tends to a finite, strictly positive constant as $N$ tends to infinity.

We will now use Donsker's invariance principle. Consider a Brownian motion $\left(W_{t}, t \geq 0\right)$ of speed $1 / d$ that starts at an $x_{0}$ of magnitude $\frac{1}{2}$, and let $\sigma_{a}=\inf \left\{t>0:\left|W_{t}\right|=a\right\}$. Then there exists a $c_{d} \in(0, \infty)$ such that, independently of the particular $x_{0}$ chosen,

$$
\mathrm{P}\left(\sigma_{1 / 3}<\sigma_{1}\right)=c_{d}
$$

- see, for example, Itô and McKean (1965). For instance, if $d \geq 3$ then $c_{d}=\left(2^{d-2}-1\right) /$ $\left(3^{d-2}-1\right)$. Thus, by path continuity and the isotropy of Brownian motion, there exists an $h_{d}>0$ such that, for all $x_{0}$ of magnitude $\frac{1}{2}$,

$$
\mathrm{P}^{x_{0}}\left(\sigma_{1 / 3}<\sigma_{1} \wedge h_{d}\right)>\frac{1}{2} c_{d}
$$

By Donsker's invariance principle and a simple compactness argument, we have that, for $n$ sufficiently large, and uniformly over all initial positions $x_{0}$ on $\delta\left(B_{n / 2}\right)$ (the boundary of $B_{n / 2}$ ), the probability that a simple random walk starting from $x_{0}$ hits $B_{n / 3}$ before leaving $B_{n}$ and before time $h_{d} n^{2}$ is at least $\frac{1}{2} c_{d}$.

Thus, by repeatedly using the strong Markov property, we find that, for $n$ sufficiently large, the simple random walk starting at $x_{0}$ on $\delta\left(B_{n / 2}\right)$ will exit $B_{n}$ after time $n^{2} h_{d} N$ with a probability of at least $\left(\frac{1}{2} c_{d}\right)^{N}$. 
This fact and (1) imply that

$$
\mu(n) \geq\left(\frac{1}{2} c_{d}\right)^{1 / n^{2} h_{d}}>1-a
$$

for $n$ sufficiently large. This completes the proof of Lemma 1.

We now fix $\varepsilon=\lambda_{1}-1>0$ and consider a simple branching process in which particles die at rate 1 and split in two at rate $1+\frac{1}{2} \varepsilon$. Alternatively, $\left(X_{t}, t \geq 0\right)$ is a birth-death process with 0 an absorbing state, i.e.

$$
\begin{aligned}
& q_{n, n+1}=n\left(1+\frac{1}{2} \varepsilon\right), \\
& q_{n, n-1}=n .
\end{aligned}
$$

It is well known that if $X_{0}=1$, then $\left(X_{t} \mathrm{e}^{-t \varepsilon / 2}, t \geq 0\right)$ is an $L^{2}$-bounded martingale. Let this bound be denoted by $K$.

Lemma 2. For all $\delta>0$, we have

$$
\mathrm{P}\left(\sup _{t>0}\left|\frac{X_{t}}{X_{0}} \mathrm{e}^{-\varepsilon t / 2}-1\right|>\delta\right)<\frac{2}{\delta} \frac{K}{\sqrt{X_{0}}} .
$$

Proof. Note that

$$
M_{t}=\frac{X_{t}}{X_{0}} \mathrm{e}^{-\varepsilon t / 2}-1
$$

is a martingale with $M_{0}=0$. Thus, for any $T>0$,

$$
\mathrm{P}\left(\inf _{t \leq T} M_{t} \leq-\delta\right) \leq \frac{\mathrm{E}\left(M_{T}^{+}\right)}{\delta}
$$

- see, for example, Ethier and Kurtz (1986, Equation 2.47). Similarly, we have

$$
\mathrm{P}\left(\sup _{t \leq T} M_{t} \geq \delta\right) \leq \frac{\mathrm{E}\left(M_{T}^{+}\right)}{\delta}
$$

and, therefore,

$$
\mathrm{P}\left(\sup _{t \leq T}\left|M_{t}\right| \geq \delta\right) \leq 2 \frac{\mathrm{E}\left(M_{T}^{2}\right)^{1 / 2}}{\delta} .
$$

We now compute

$$
\mathrm{E}\left(M_{T}^{2}\right)=\frac{1}{X_{0}^{2}}\left\|X_{T} \mathrm{e}^{-\varepsilon T / 2}-X_{0}\right\|_{2}^{2},
$$

where $\|\cdot\|_{2}$ denotes the $L^{2}$-norm. We write $X_{t}$ as a sum of $X_{0}$ independent and identically distributed processes $Y_{t}^{(i)}, 1 \leq i \leq X_{0}$, which have the same rates as $X_{t}$ and initial state 1 . Thus,

$$
\mathrm{E}\left(M_{T}^{2}\right)=\frac{1}{X_{0}^{2}}\left\|\sum_{i=1}^{X_{0}}\left(Y_{T}^{(i)} \mathrm{e}^{-\varepsilon T / 2}-1\right)\right\|_{2}^{2} .
$$

By using the independence of $Y_{T}^{(i)}, 1 \leq i \leq X_{0}$, we find that

$$
\mathrm{E}\left(M_{T}^{2}\right)=\frac{1}{X_{0}^{2}} X_{0}\left\|Y_{T}^{(1)} \mathrm{e}^{-\varepsilon T / 2}-1\right\|_{2}^{2} \leq \frac{K^{2}}{X_{0}} .
$$

This completes the proof of Lemma 2. 
Now, pick an $a>0$ such that

$$
\frac{(1+\varepsilon)(1-a)}{1+\frac{1}{2} \varepsilon}>1 .
$$

We choose $N_{0}$ satisfying Lemma 1 for this $a$. We now go back to the eigenfunction $f \equiv f_{N_{0}}$ corresponding to the largest eigenvalue $\mu(n)$ of the subprobability matrix for the simple random walk, with Dirichlet boundary conditions, on $B \equiv B_{N_{0}}$. By the Perron-Frobenius theorem, $f$ is strictly positive on $B$ and, thus, has a minimum value $m>0$ and, for all $x$ in $B$ and $N_{1}$, satisfies

$$
\frac{f(x) N_{1}+1}{f(x) N_{1}} \leq \frac{m N_{1}+1}{m N_{1}} .
$$

Pick a $\delta>0$ small enough that

$$
\frac{(1+\varepsilon)(1-a)}{1+\frac{1}{2} \varepsilon} \frac{1-\delta}{1+\delta}>1 .
$$

Then there exists an integer $N_{1}$ large enough that

$$
\frac{(1+\varepsilon)(1-a)}{1+\frac{1}{2} \varepsilon} \frac{1-\delta}{1+\delta}>\frac{m N_{1}+1}{m N_{1}} .
$$

Lemma 3. There exists a system $\left(X_{t}^{x}, t \geq 0\right)_{x \in B}$ of identically distributed birth-death processes, with rates $q_{i, j}$, such that if $X_{0}^{x}=\xi_{0}(x)=\left\lceil f(x) N_{1}\right\rceil$ for all $x$ in $B$, then the following coupling holds:

$$
X_{t}^{x} \leq \xi_{t}(x) \text { for all } t \leq \tau \text { and all } x \in B,
$$

where

$$
\tau=\inf \left\{s: \text { there exists an } x \in B \text { such that } \frac{X_{s}^{x}}{X_{0}^{x}} \mathrm{e}^{-\varepsilon s / 2} \notin(1-\delta, 1+\delta)\right\} .
$$

Proof. We will explicitly construct $\left(X_{t}^{x}, t \geq 0\right)_{x \in B}$ from the process $\left(\xi_{t}(x), t \geq 0\right)_{x \in B}$, thus providing a coupling of the two processes.

Let $\left(Y_{t}^{x}(n), t \geq 0\right)_{x \in B, n \geq 1}$ be mutually independent Poisson processes, independent of $\left(\xi_{t}(x): t \geq 0\right)_{x \in B}$ and such that $Y_{t}^{x}(n)$ has rate $n$. If there is a death at time $t$ for $\xi_{t}(x)$ and if $X_{t}^{x} \leq \xi_{t}(x)$, then there is a death at $t$ for $X_{t}^{x}$ with probability $X_{t}^{x} / \xi_{t}(x)$. If $X_{t}^{x}=n>\xi_{t}(x)$ and there is a birth at $t$ for the Poisson process $Y_{t}^{x}(n)$, then there is a death at $t$ for $X_{t}^{x}$.

For births, we use a similar method. Let $\left(Z_{t}^{x}(n), t \geq 0\right)_{x \in B, n \geq 1}$ be mutually independent Poisson processes, independent of $\left(\xi_{t}(x), t \geq 0\right)_{x \in B}$ and such that $Z_{t}^{x}(n)$ has rate $n\left(1+\frac{1}{2} \varepsilon\right)$. If there is a birth at $x$ at time $t$ for $\xi_{t}(x)$ and if $\left(1+\frac{1}{2} \varepsilon\right) X_{t-}^{x} \leq \lambda_{1} \sum_{y \sim x} \xi_{t}(y) /(2 d)$, there is a birth at the same time for $X_{t}^{x}$ with probability

$$
\frac{2 d\left(1+\frac{1}{2} \varepsilon\right) X_{t-}^{x}}{\lambda_{1} \sum_{y \sim x} \xi_{t}(y)}
$$

If $\left(1+\frac{1}{2} \varepsilon\right) X_{t-}^{x}=n>\lambda_{1} \sum_{y \sim x} \xi_{t}(y) /(2 d)$ and there is a birth at time $t$ for the process $Z_{t}^{x}(n)$, then there is a birth at the same time for $X_{t}^{x}$.

The condition that $\xi_{t}(x) \geq X_{t}^{x}$ for all $x \in B$ can evidently never be violated by a death (recall that $X_{0}^{x}=\xi_{0}(x)$ for all $x \in B$ ), so it remains to check that, for $t<\tau$, the domination relation holds for births as well. 
Assume that $t<\tau$ and $x \in B$, and that the upwards flip rate for $X_{t}^{x}$ is $\left(1+\frac{1}{2} \varepsilon\right) X_{t-}^{x}$ while that for $\xi_{t}^{x}$ at time $t$ is

$$
(1+\varepsilon) \sum_{y \sim x} \frac{\xi_{t-}(y)}{2 d} .
$$

By hypothesis, $\xi_{t-}(y) \geq X_{t-}^{y}$ for each relevant $y$, so this flip rate exceeds

$$
(1+\varepsilon) \sum_{y \sim x} \frac{X_{t-}^{y}}{2 d} .
$$

By the fact that $t<\tau$, the rate (2) is greater than

$$
(1+\varepsilon) \sum_{y \sim x}\left\lceil f(y) N_{1}\right\rceil \mathrm{e}^{\varepsilon t / 2} \frac{1-\delta}{2 d} \geq(1+\varepsilon)\left\lceil\sum_{y \sim x} f(y) N_{1}\right\rceil \mathrm{e}^{\varepsilon t / 2} \frac{1-\delta}{2 d}
$$

and, by Lemma 1, the right-hand side of (3) is greater than

$$
(1+\varepsilon)\left\lceil(1-a) 2 d f(x) N_{1}\right\rceil \mathrm{e}^{t \varepsilon / 2} \frac{1-\delta}{2 d} \geq(1+\varepsilon)(1-a) 2 d f(x) N_{1} \mathrm{e}^{t \varepsilon / 2} \frac{1-\delta}{2 d} .
$$

Recall that $N_{1}$ has been chosen so that

$$
\frac{(1+\varepsilon)(1-a)}{1+\frac{1}{2} \varepsilon} \frac{1-\delta}{1+\delta}>\frac{m N_{1}+1}{m N_{1}} \geq \frac{f(x) N_{1}+1}{f(x) N_{1}} .
$$

Thus, for all $x$ in $B$,

$$
(1+\varepsilon)(1-a) f(x) N_{1} \mathrm{e}^{t \varepsilon / 2}(1-\delta) \geq\left(1+\frac{1}{2} \varepsilon\right)\left(1+f(x) N_{1}\right) \mathrm{e}^{t \varepsilon / 2}(1+\delta) .
$$

In turn, the right-hand side of (4) is greater than

$$
\left(1+\frac{1}{2} \varepsilon\right)\left\lceil f(x) N_{1}\right\rceil \mathrm{e}^{t \varepsilon / 2}(1+\delta) \geq\left(1+\frac{1}{2} \varepsilon\right) X_{t-}^{x},
$$

where the last inequality again comes from the fact that $t \leq \tau$. This shows that the domination conditions cannot be violated for $t<\tau$, and concludes the proof of Lemma 3 .

We now conclude the proof of Theorem 1. Assume that $\xi_{0}(x)=\left\lceil f(x) N_{1}\right\rceil$ for every $x \in B$ and let $\mathcal{A}$ be the event

$$
\mathcal{A}=\left\{\text { there exist } t>0 \text { and } x \in B \text { such that } \xi_{t}(x)<(1-\delta)\left\lceil f(x) N_{1}\right\rceil \mathrm{e}^{\varepsilon t / 2}\right\} .
$$

Note, by Lemma 3, that the intersection of the events $\{\tau=\infty\}$ and $\mathcal{A}$ is empty. Thus,

$$
\mathrm{P}(\mathcal{A}) \leq \mathrm{P}(\tau<\infty) \leq \sum_{x \in B} \frac{2}{\delta} \frac{K}{\sqrt{\left\lceil f(x) N_{1}\right\rceil}},
$$

where the second inequality comes from Lemma 2. Since $f$ is strictly positive on $B$, we may pick $N_{1}$ large enough that

$$
\min _{x \in B}\left\lceil f(x) N_{1}\right\rceil \geq 4 \frac{K^{2}}{\delta^{2} \alpha^{2}}|B|^{2}
$$

and, so,

$$
\mathrm{P}(\mathcal{A}) \leq \alpha .
$$

This concludes the proof of Theorem 1 for small $\delta$. The theorem for all $\delta \in(0,1)$ follows naturally. 


\section{Acknowledgements}

RBS thanks Enrique Andjel for numerous helpful discussions. We also thank an anonymous referee whose careful reading helped improve the presentation of the paper.

\section{References}

Aldous, D. AND Fill, J. (2003). Reversible Markov chains and random walks on graphs. Work in progress. Drafts of chapters available at http://www.stat.berkeley.edu/users/aldous/.

ENGLÄNDER, J. AND KyPRIANOU, A. E. (2004). Local extinction versus local exponential growth for spatial branching processes. Ann. Prob. 32, 78-99.

ENGLÄNDER, J. AND PINSKY, R. G. (1999). On the construction and support properties of measure-valued diffusions on $D \subseteq \mathbb{R}^{d}$ with spatially dependent branching. Ann. Prob. 27, 684-730.

Ethier, S. N. And Kurtz, T. G. (1986). Markov Processes. Characterization and Convergence. John Wiley, New York.

Itô, K. And McKean, H. P. (1965). Diffusion Processes and Their Sample Paths. Springer, Berlin.

Kesten, H. And van den Berg, J. (2000). Asymptotic density in a coalescing random walk model. Ann. Prob. 28, 303-352.

Liggett, T. M. (1985). Interacting Particle Systems, Springer, New York.

Liggett, T. M. (1999). Branching random walks on finite trees. In Perplexing Problems in Probability (Progress Prob. 44), eds M. Bramson and R. Durrett, Birkhäuser, Boston, MA, pp. 315-330.

Neuhauser, C. (1992). Ergodic theorems for the multitype contact process. Prob. Theory Relat. Fields 91, 467-506.

Pemantle, R. And Stacey, A. M. (2001). The branching random walk and contact process on Galton-Watson and nonhomogeneous trees. Ann. Prob. 29, 1563-1590. 